\title{
Quantum Turbulence at Very Low Temperatures: Status and Prospects
}

\author{
D. Charalambous*, P.C. Hendry*, P.V.E. McClintock* and L. Skrbek ${ }^{\dagger}$ \\ *Department of Physics, University of Lancaster, Lancaster LAI 4YB, UK \\ $\dagger$ Joint Low temperature Laboratory, Institute of Physics ASCR and Faculty of Mathematics and Physics, Charles \\ University, V Holešovičkách 2, 18000 Prague, Czech Republic
}

\begin{abstract}
The theory of how turbulent energy decays via a Richardson cascade is well-established for classical fluids, and it also seems to apply to the case of so-called co-flowing He II turbulence in the range from the superfluid transition temperature $T_{\lambda}$ down to $\sim 1 \mathrm{~K}$, where its behaviour is similar to that of a classical fluid. For pure superfluids, e.g. He II in the $\mathrm{mK}$ range or ${ }^{3} \mathrm{He}-\mathrm{B}$ in the $\mu \mathrm{K}$ range, where the normal fluid density $\rho_{n}$ is near zero, the mode(s) through which quantum turbulence (QT) might decay have been much less clear because of the absence of viscosity to dissipate the turbulent energy on small length scales. Recent advances made in the theory of QT in this $T \rightarrow 0$ limit are consistent with such experimental evidence as is available, but new experiments supported by new techniques for the production and detection of QT are urgently required. The experimental situation is reviewed and prospects for further advances are considered.
\end{abstract}

Keywords: helium, superfluidity, turbulence, oscillators PACS: $47.27 . \mathrm{Cn}, 47.37 .1 \mathrm{q}, 67.40 . \mathrm{Vs}, 47.15 . \mathrm{Cb}$

\section{INTRODUCTION}

Quantum turbulence (QT) $[1,2]$ in a superfluid $\left({ }^{4} \mathrm{He}\right.$ or $\left.{ }^{3} \mathrm{He}-\mathrm{B}\right)$ can be thought of as a seemingly disordered collection of quantized vortex lines. The vortex lines themselves have been well-understood almost since their original discovery $[3,4]$ half a century ago. To reach an understanding of their collective motion in QT, however, represents a problem in complexity where major challenges remain to be tackled. It must be emphasized that the vortices are the components of QT. One can no more expect to predict the properties of QT from an understanding of these components than one could e.g. predict second sound or the other superfluid properties of liquid ${ }^{4} \mathrm{He}$ from a detailed knowledge of the ${ }^{4} \mathrm{He}$ atom; likewise, neither could one infer the periodic table, or chemistry, from a detailed knowledge of protons, neutrons and electrons. In each case, there are emergent phenomena that could hardly have been anticipated from an understanding of the microscopic components of the system. Note also that, in reality, and contrary to their appearance, the arrangement of vortex lines in QT is not random.

Below its superfluid transition temperature $T_{\lambda}$, liquid ${ }^{4} \mathrm{He}$ of density $\rho$ can be regarded [5] as a mixture two components: a normal fluid component with viscosity, carrying the whole entropy of the liquid, and an inviscid superfluid carrying no entropy, with densities $\rho_{n}$ and $\rho_{s}$ respectively such that $\rho_{n}+\rho_{s}=\rho$. Quantum mechanics places severe restrictions on the motion of the superfluid, in that the circulation is quantized

$$
\oint \mathbf{v}_{s} \cdot \mathbf{d} \ell=h / m_{4}
$$

where $\mathbf{v}_{s}$ is the fluid velocity, $m_{4}$ is the helium atomic mass and the integral is taken around a loop enclosing the vortex core; there is no such restriction on motions in the normal fluid component, which are classical. In the case of ${ }^{3} \mathrm{He}$, the $m_{4}$ is replaced by $2 m_{3}$, the mass of a Cooper pair.

The relative proportions of the two fluids are such that the normal fluid density $\rho_{n} / \rho=1$ at $T=T_{\lambda}$ and $\rho_{n} / \rho=0$ at $T=0$; correspondingly, the superfluid density $\rho_{s} / \rho=0$ at $T=T_{\lambda}$ and $\rho_{s} / \rho=1$ at $T=0$. In a typical experiment, QT is created by flow or by motion of an object in the liquid. In what follows, we will assume conventional isothermal flow; we will not consider superfluid/normal fluid counterflow (which, although often discussed in the literature, is actually a rather specialised kind of flow, peculiar to superfluids). Because the vortex lines are metastable states of the liquid, they may be expected to decay away with time. The main aim of the theory is to identify the underlying decay mechanisms, explore the corresponding physics, and characterise the form and timescale of the decay for comparison with experiment. As we will see, the theoretical situation is much clearer in the temperature range where the normal fluid density $\rho_{n}$ is appreciable, say for $1<T<T_{\lambda}=2.17 \mathrm{~K}$. The main unsolved questions relate to the $T \rightarrow 0$ limit, by which we mean the temperature range in which $\rho_{n} \simeq 0$ so that the effect of viscous dissipation is negligible. 
The aim of this paper is to introduce QT in a manner accessible to non-specialists, to outline succinctly what is already understood, and to identify the major challenges still to be tackled. In particular, we describe the emergent theoretical picture describing QT in the $T \rightarrow 0$ limit, consider some of the experiments carried out to date in this temperature range, and discuss the requirements of the new generation of experiments now needed to test the theory. Our emphasis will be on experiments, but first we consider the theoretical background in order to set the context of the work.

\section{THEORETICAL BACKGROUND}

\section{Classical turbulence}

In order to appreciate the problems presented by QT, it is first necessary to understand the accepted picture of classical turbulence (CT) $[6,7]$ and the manner in which it decays. The flow of the fluid is governed by the NavierStokes equation [8]

$$
\frac{\partial \mathbf{v}}{\partial t}+(\mathbf{v} . \nabla) \mathbf{v}=-\frac{1}{\rho} \nabla p+v \nabla^{2} \mathbf{v}
$$

where $\mathbf{v}$ is the flow velocity, $p$ is the pressure, $\rho$ is the density, and $v$ is the kinematic viscosity. The ratio of the nonlinear inertial term $(\mathbf{v} . \nabla) \mathbf{v}$ to the dissipative term $v \nabla^{2} \mathbf{v}$ gives the Reynolds number $\operatorname{Re}=\ell u / v$. Here, $u$ and $\ell$ are respectively a characteristic velocity and length describing the flow. In turbulent flows, Re is large and so the effect of dissipation can be ignored.

In typical flows, energy is fed into the turbulence at large length scales determined e.g. by the dimensions of a flow tube or the mesh size of a grid. Because the nonlinear term in (2) couples together motion on different length scales, turbulent energy flows without dissipation towards smaller and smaller length scales. Eventually the little eddies become so small that $\operatorname{Re} \sim 1$ : the dissipative term in (2) then becomes important, and the energy is dissipated by viscosity. Within the inertial range of this Richardson cascade, the spectral energy density distribution is described by the Kolmogorov spectrum

$$
E(k)=C \varepsilon^{2 / 3} k^{-5 / 3}
$$

where $k$ is the wave number of the eddies, $E(k)$ is the energy per unit mass contained in eddies of wavenumber between $k$ and $k+d k$, the Kolmogorov constant $C$ is of order unity, and $\varepsilon$ determines the rate at which energy flows down the cascade. Note that the fluid dynamics community commonly uses a notation where the turbulent energy is specified per unit mass, so it is of dimension $\mathrm{m}^{2} / \mathrm{s}^{2}$.
Here we take no account of intermittency, despite its being a keyword of today's conventional turbulence research. We note that there is experimental evidence [9] strongly suggesting that intermittency occurs in QT as well, at least in the case of co-flowing He II turbulence generated between counterrotating discs [9]. Although its role in QT has not yet been widely recognized, there is little doubt that intermittency will become an important issue in future QT studies.

\section{Quantum turbulence}

We first consider QT at finite $T$. In mechanically generated co-flowing He II, the normal and superfluid components move together on large length scales, being strongly coupled by the action of the mutual friction force. The latter arises because, although vortex lines consist of an ordered flow in the superfluid component, the vortex cores around which the flow rotates are part of the normal fluid component ${ }^{1}$. Consequently, flows of this kind e.g. grid turbulence are characterised by a hydrodynamics that is essentially classical on large length scales, closely resembling conventional turbulence. On smaller scales, however, the quantum condition (1) will dominate and non-classical behaviour is then to be anticipated. In particular, the decay of the vortex tangle is expected to be driven by line reconnections [10], as sketched in Fig. 1. They create relatively sharp cusps in the reconnected lines, and the fast self-induced superflow velocity causes rapid motion of the vortex core through the viscous normal fluid component, leading to line shrinkage. Experiments above $1 \mathrm{~K}$ have studied QT created between two

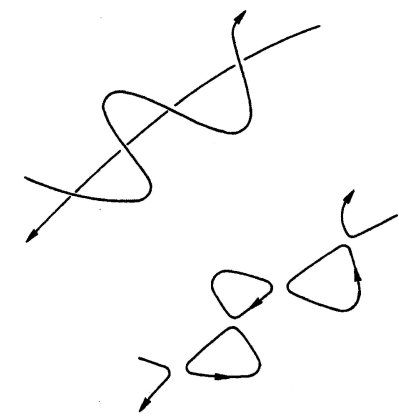

FIGURE 1. Sketch showing how quantized vortices are believed to reconnect when they cross, after Schwarz [10].

\footnotetext{
${ }^{1}$ In ${ }^{3} \mathrm{He}-\mathrm{B}$, considered later, the cores of the quantized vortices are macroscopic objects about hundred times larger than those of $\mathrm{He}$ II vortices, with complex structure that undergoes phase transitions. Nevertheless, the concept of the mutual friction force between normal and superfluid components holds generally.
} 
counter-rotating disks [9], and the decay of QT created by a grid drawn once through a column of He II $[11,12]$. In each case, the inferred energy distribution of the turbulence was consistent with the Kolmogorov energy spectrum (3) [13]. At least in this respect, therefore, QT is (perhaps unexpectedly) similar to classical turbulence.

In the $T \rightarrow 0$ limit, the absence of normal fluid component means that reconnections are no longer dissipative through this mechanism (though some small dissipation due to this process may still be expected). So, given that the cascade to smaller scales cannot be terminated by viscous damping, what happens? We can even wonder whether the QT will ever decay. Physical intuition suggests that it must in fact decay, given that vortices represent metastable states of the liquid - and experiments (see below) confirm this tentative conclusion. The theory of QT in the zero $T$ limit and the decay mechanism in this regime $[1,15,16,17,18,19,20]$ is due especially to Vinen, Barenghi, Brachet, Svistunov, Tsubota and others. We draw attention to the ever-growing role of numerical studies in such investigations. They

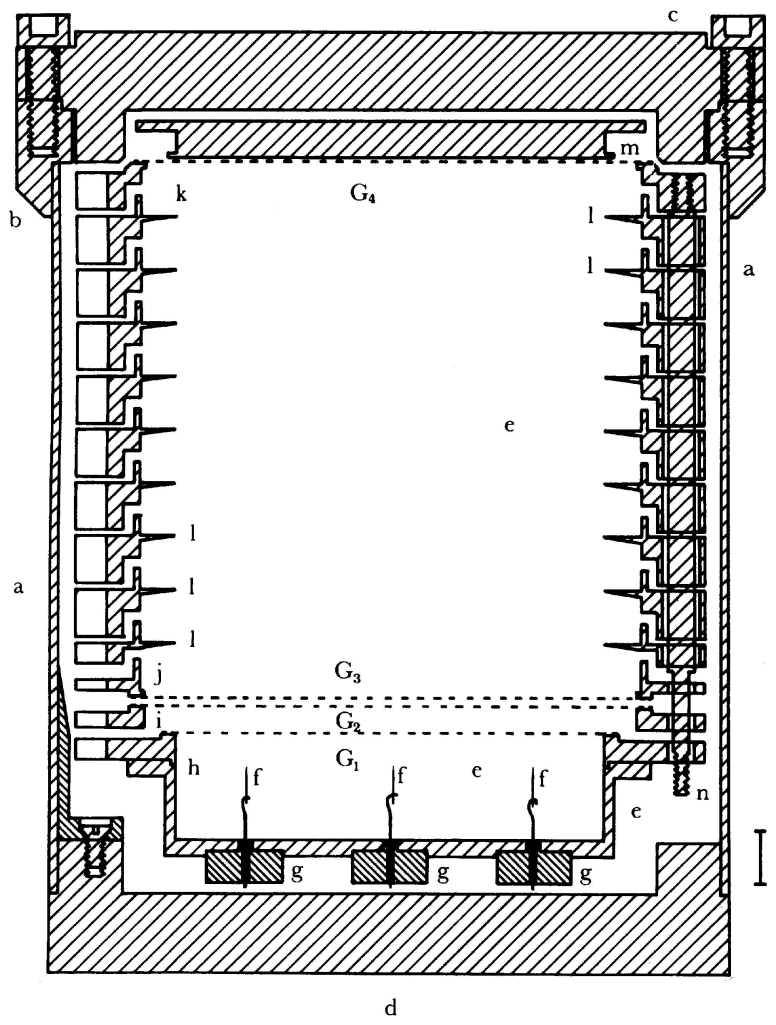

FIGURE 2. Schematic diagram of cell used for measurement of the Landau critical velocity [14]. The field emission tips $f$ were used to inject negative ions, whose drift velocity across the space between grids $G_{3}$ and $G_{4}$ was then measured. The $10 \mathrm{~mm}$ vertical bar outside the cell on the lower right-hand side is to indicate the scale. can now be based on a full Biot-Savart simulation of the motion of vortex filaments [18, 21], as well as on the non-linear Schrödinger equation (Gross-Pitaevskii equation) $[22,23]$. These studies follow the influential work of Schwarz [10] who recognized the importance of reconnections, but in an era when the available computer power forced him to limit his simulations to the so-called local induction approximation, thus neglecting any possibility of non-local interaction. Thus e.g. stretching of vortex lines, which is believed to play a central role in classical turbulence, could not be included.

The picture that is emerging involves a Richardson cascade from the largest length scales to successively smaller scales. It cannot end in viscous dissipation, as in the classical case, but it is probably followed by a Kelvin wave cascade. Reconnections generate a high density of Kelvin waves (vortex waves) on the lines, which mutually interact to generate Kelvin waves of successively higher frequency. Those of sufficiently high frequency can radiate phonons, thus terminating the cascade. What experimental evidence is there to support this general picture?

\section{Early results}

Interest in the decay of QT preceded the development of the theory. The experimental determination of the Landau critical velocity for roton creation [14] involved the use of the large experimental cell shown in Fig. 2. Negative ions were injected from the field emission tips $\mathrm{f}$, and their transit time across the relatively low electric field region between grids $\mathrm{G}_{3}$ and $\mathrm{G}_{4}$ was measured. It is known that charged vortex rings and QT are created in the region of very high electric field near field emission tips. Negative ions are trapped on vortex lines so that, in the absence of a QT decay mechanism, a high density of spacecharge would have built up within the cell and would have prevented the experiment from working. That this did not happen [14] demonstrated that a QT decay mechanism must exist even in the absence of normal fluid component.

Fig. 3 illustrates a preliminary experiment [24] to investigate the decay of QT in superfluid ${ }^{4} \mathrm{He}$ in the $T \rightarrow 0$ limit. A fine-mesh grid, stretched to its yield point on a circular holder, is driven on-resonance by an electrostatic field. Because of the high $Q$ of the oscillator, and the fact that it is vibrating in a dissipation-free superfluid, the amplitude rises until the critical velocity for QT generation is attained. After the grid has been driven for a few seconds, the drive is switched off. When a pulse of negative ions is passed through the QT that has been created, some of the ions get trapped on the vortices, leading to an attenuated signal at the collector. By propagating a se- 
quence of such pulses, the recovery of the signal can be observed, corresponding to the decay of the QT cloud as shown in Fig. 4. Although direct quantitative comparison of the results of this experiment with the emerging theoretical or computational models is difficult, it clearly illustrates the decay of QT on a timescale of seconds.

\section{EXPERIMENTS ON QT AT VERY LOW T}

\section{Techniques for creating QT}

In addition to the vibrating grid mentioned above, several other techniques have also been used for creating QT in the low $T$ limit, illustrated in Fig. 5.

The most widely used of these is the oscillating wire loop (top) [25, 26, 27]. The loop is formed from a single NbTi superconducting filament etched out of the copper matrix of standard magnet wire, typically $\mathrm{x}-\mathrm{y} \mu \mathrm{m}$ in diameter. A magnetic field is applied parallel to its legs. When an oscillatory current is passed through the loop, it responds to the resultant force as shown, moving in the direction perpendicular to the magnetic field and the wire. The induced voltage arising from the movement can be measured directly, there being no voltage due to the current in the static wire on account of its superconductivity (and we ignore the possibility of flux motion within the wire, associated with its movement).

At small driving amplitudes the response is usually linear, corresponding to laminar flow. Under these conditions the superfluid makes no contribution to the damping, but just provides an added hydrodynamic mass (see below). When the driving amplitude is such that a critical velocity is attained there is a sudden change in the re-

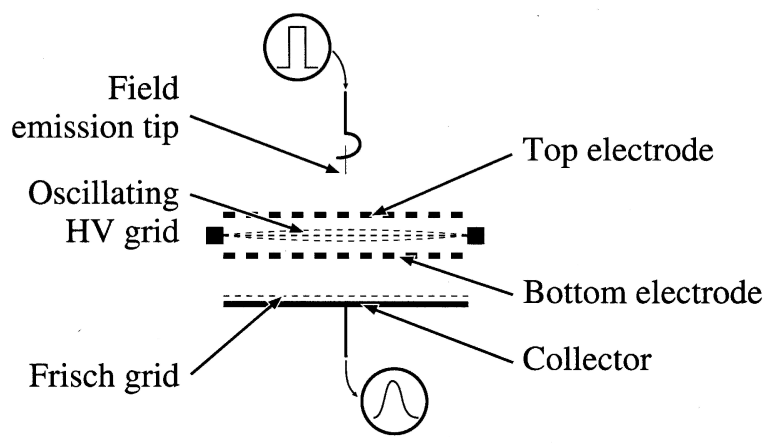

FIGURE 3. Schematic diagram showing the apparatus used for the preliminary experiment seeking evidence of QT decay at very low temperatures [24]. The electrode structure was immersed in isotopically pure He II with the cell illustrated in Fig. 2.

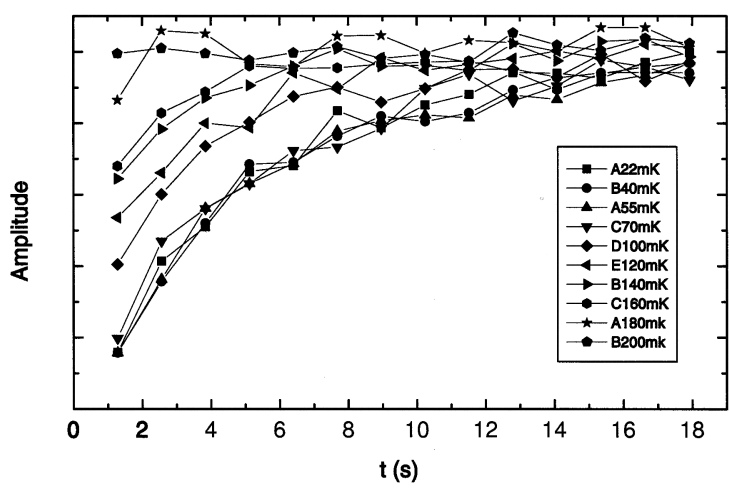

FIGURE 4. Amplitudes of a sequence of negative ion pulses after passage through the QT cloud [24]. The amplitude increases with time because the QT decays, reducing the extent of ion trapping on quantized vortices.

sponse, attributable to the creation of QT ${ }^{2}$. In the crossover regime, a variety of interesting phenomena can appear, including hysteresis and intermittent switching between laminar and turbulent flow.

The oscillating microsphere (Fig. 5, second from top) behaves in many respects rather similarly [28, 29, 30, 31]. The magnetic sphere is levitated in He II between $\mathrm{Nb}$ electrodes. It carries an electrostatic charge, and it can therefore be driven by an applied oscillatory electric field. The resultant oscillations can be detected and measured. Again, investigation of the variation of the response amplitude with the driving force reveals a welldefined critical velocity at which there is a marked change in behaviour from linear response (laminar flow) to a much slower increase in response with drive (because of the creation of QT): see Fig. 6.

A recent innovation by Bradley et al [32] (Fig. 5, third from top) has been the creation of QT in ${ }^{3} \mathrm{He}-\mathrm{B}$ by means of a grid mounted on a loop of superconducting wire. The grid material is identical to that used in the earlier work in He II [24, 33, 34], but it is moved magnetically rather than electrostatically. The authors report convincing evidence (see below) of QT production in ${ }^{3} \mathrm{He}-\mathrm{B}$ when a critical velocity of the grid is exceeded.

A very recent innovation is the use of an oscillating quartz tuning fork [35]. The legs of the fork (Fig. 5, bottom) are about $3.4 \mathrm{~mm}$ in length, each with a crosssection of $400 \times 400 \mu \mathrm{m}^{2}$. They oscillate in antiphase at a frequency of $\sim 30 \mathrm{kHz}$. Preliminary studies suggest that the behaviour of the fork in superfluid is generally similar to that of other vibrating objects in that it creates QT once

\footnotetext{
${ }^{2}$ In ${ }^{3} \mathrm{He}-\mathrm{B}$ there is also a critical threshold associated with reaching the pair-breaking velocity [26]. The Landau critical velocity for roton creation in He II [14] is many orders of magnitude larger than those at which QT is generated, and can be ignored in the present context.
} 
a critical velocity has been exceeded. It is characterised by robustness, small size, and a high $Q$ of up to $10^{5}$, and there may be other advantages. These devices are under active investigation in Helsinki, Košice, Lancaster and Prague in terms of their potential for thermometry and particle detection, as well as for QT creation, both in $\mathrm{He}$ II and superfluid phases of ${ }^{3} \mathrm{He}$.
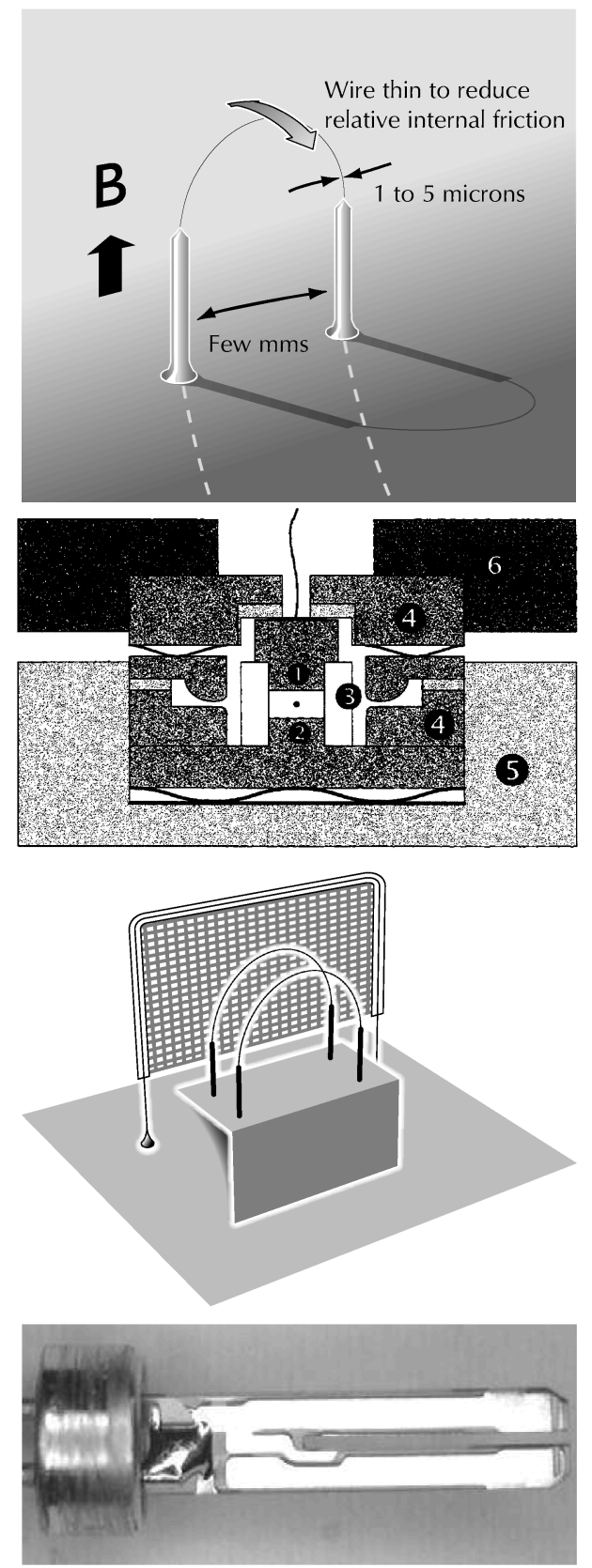

FIGURE 5. Other techniques used for creation of QT in superfluid ${ }^{4} \mathrm{He}$ and ${ }^{3} \mathrm{He}$ at very low $T$. From the top: vibrating wire loop [25, 26, 27]; oscillating levitated sphere [28, 29, 30, 31 ]; vibrating grid, on wire loop (with two additional loops as detectors) [32]; vibrating quartz fork [35].
All of the techniques shown in Figs. 3 and 5 involve the oscillation of a submerged object of some kind within the superfluid. Despite obvious cryogenic advantages this is not, in fact, ideal as a method of creating QT (see Discussion below) in that one aims to create a well-defined turbulent flow that can be approximated as homogeneous and isotropic in order to compare it with available theoretical models.

\section{Hydrodynamic mass in a superfluid}

The superfluid is inviscid and, provided that there are no free surfaces on which waves can be created, it causes no drag on an object moving at low velocity. Nonetheless, there are inertial effects associated with the need to push the superfluid out of the way. This is the origin of the classical hydrodynamic mass enhancement of

$$
\Delta M=\beta V \rho
$$

where $\beta$ is a geometrical constant, $V$ is the volume of the moving object, and $\rho$ is the density of the fluid. The dynamics of a moving object in the superfluid in the $T \rightarrow 0$ limit are therefore identical to what they would be in a vacuum, except for the modified effective mass. Experiments with negative ion bubbles [36] confirm this picture.

For oscillating material objects submerged in He II, however, there are indications $[37,38]$ that the effective mass is enhanced by more than just the classical $\Delta M$, even within the linear regime of low oscillatory amplitude. In the case of the oscillating grid, the constant $\beta$ cannot reliably be evaluated because of the ill-defined though approximately rectangular cross-section of the grid wires, but Charalambous et al [37] arrive at this conclusion through investigation of the density (pressure) dependence of the resonant frequency. The wire used by Yano et al [38] is smooth and circular in cross-section, so that the value of $\beta=1$ for an infinite cylinder can be assumed. The radius of the wire calculated from the measured hydrodynamic mass is significantly larger than the actual radius as determined by electron microscopy, i.e. the effective mass is larger than expected. It seems extremely likely that the physics underlying the anomalous mass enhancement is the same in both experiments $[37,38]$. The origin of the effect has yet to be established, but one possibility is that surfaces exposed to superfluid become covered in a "fur" of small vortex loops that can then add [39] to the inertial effective mass. The general picture would be qualitatively similar to that considered by Kusmartsev [40] in trying to account for critical velocities in the flow of rotating superfluid ${ }^{4} \mathrm{He}$. He envisaged the creation by fluctuations of a plasma of halfvortex rings at the wall. In terms of this picture, the con- 
cept of remanent vorticity [41], consisting of a few relatively long vortices pinned to protuberances, must be supplemented by the idea of the dense array of microscopic loops. But many problems need to be resolved (e.g. the stability of pinned vortex half-rings) before any firm conclusion can be drawn.

The seemingly anomalous effective mass of objects oscillating in the superfluid does not bear directly on QT as such, but the underlying physics may be highly relevant to the critical velocity that must be exceeded to initiate QT creation.

\section{Techniques for detecting QT}

Experiments on QT in He II above $1 \mathrm{~K}[11,12]$ have used the attenuation of second sound to detect the vortices, almost universally. It is an extremely sensitive technique: using a resonant cavity, it can even enable the detection of individual vortex lines. Unfortunately, however, it is applicable neither in the low $T$ range for He II because second sound (involving antiphase oscillation of the normal and superfluid components) cannot exist in the absence of normal fluid component, nor in superfluid phases of ${ }^{3} \mathrm{He}$ where second sound is heavily damped.

Most experiments on QT in He II in the $T \rightarrow 0$ limit have detected its creation indirectly, by observing the oscillatory dynamics of the moving object. In particular, the response as a function of the amplitude of the driving force usually undergoes a marked change at the transition between laminar flow and QT creation: see e.g. the oscillating microsphere data of Fig. 6. Although this approach provides evidence of QT production, it does not allow comparison with the theory described above. Exceptions include the preliminary ${ }^{4} \mathrm{He}$ experiment [24] where ion trapping was used as a detection method.

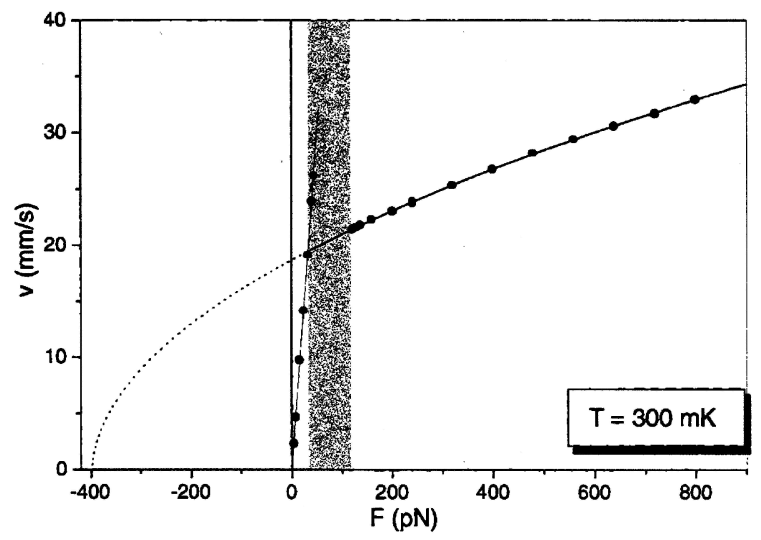

FIGURE 6. Plot of response as a function of forcing amplitude for the magnetically levitated microsphere [30].
QT in ${ }^{3} \mathrm{He}$ can be detected by application of the powerful technique of nuclear magnetic resonance (NMR). Indeed, the temperature induced transition from laminar to turbulent flow in ${ }^{3} \mathrm{He}-\mathrm{B}$ at $\sim 0.6 T_{c}$ was observed by NMR in a rotating sample when one or few vortex seed loops were injected into the originally vortex-free Landau state [42]. However, the NMR technique used is sensitive mainly to the large-scale counterflow rather than to the signal from vortex cores; and its use at lower temperatures becomes increasingly difficult. In experiments by Bradley et al [32] QT in ${ }^{3} \mathrm{He}-\mathrm{B}$ generated by the electromagnetically oscillating grid (see above) was detected as a decrease in damping sensed by the nearby vibrating wire: the underlying physics is based on Andreev scattering (the incoming quasiparticle is back-scattered as a quasihole and vice versa) of ballistically propagating quasiparticles. The Andreev scattering channel appears through Galilean modification of the quasiparticle dispersion relation due to circulating superflow around the vortex core [43].

\section{DISCUSSION}

Several experiments show that QT can be created at low $T$, and there are two $[24,32]$ which clearly demonstrate that it decays on a timescale of several seconds. It has not yet, however, been possible to make a detailed comparison with theory. The main problem is that submerged oscillating objects create QT that is not formed at a welldefined initial length scale, because the object repeatedly traverses the QT that it has created, thus chopping it up into ill-defined smaller elements. The other problems are that the QT is not homogeneous: it may stay localised near the oscillating object, so that its density then decreases sharply with distance. Another source of inhomogeneity is that flow across the moving object often occurs at different speeds in different positions. A further complication is that the transition to turbulence due to submerged oscillating objects represents a problem which is as yet only partly understood, even for classical viscous flows. Experiments with flows due to e.g. a transversally oscillating cylinder [44], or an oscillating grid in water [45], displayed very rich and complex flow phenomena. This makes comparison of classical and quantum flows, and the problem of understanding the latter, extremely difficult.

The ideal QT experiment would somehow emulate the techniques used earlier above $1 \mathrm{~K}$ [11], in which a grid was drawn once through a column of liquid to create well-characterised homogeneous QT which was then detected with enormous sensitivity by use of second sound. For obvious reasons, this is extremely difficult to accomplish at very low $T$. QT creation by linear mechanical movement must be accomplished without 
frictional heating - a considerable technical challenge and, as mentioned above, second sound cannot be used for detection because there is no normal fluid component.

Efforts to create the necessary mechanism for a drawn grid are currently in progress. To avoid friction, the grid is to be moved on magnetic bearings. The result is the levitated, superconducting, linear, stepper motor ${ }^{3}$ sketched in Fig. 7. It is hoped that this device will avoid many of the disadvantages of the earlier oscillatory QT creation methods.

New techniques will also be required to detect the QT. There are two problems associated with the use of negative ions. First, although the capture cross-section in He II has not yet been measured in the $T \rightarrow 0$ limit, it is certainly very small [46] and probably highly dependent on electric field. So ions can only be suitable for the detection of QT at relatively high density and will probably not allow coverage of the wide range of densities needed for a detailed comparison with experiment. Secondly, they must be to some extent invasive probes in that any electric field, including their own spacecharge field, will apply a force to the QT, an effect that could be significant under certain circumstances.

The use of ions for QT detection in the superfluid phases of ${ }^{3} \mathrm{He}$ seems even more difficult, in view of a much larger size of the vortex cores leading to smaller substitution energy and presumably a smaller capture cross-section. The use of NMR and especially the novel detection technique based on Andreev scattering (see above) seem promising here. There is a clear call for complementary experiments probing comparable flows in $\mathrm{He}$ II and ${ }^{3} \mathrm{He}$ in the $T \rightarrow 0$ limit.

Further detection techniques currently under consideration include the use of: (a) calorimetry, which is sensitive but where it will be difficult to analyse the data for comparison with the theory; (b) tracers, e.g. excited He II molecules; and (c) miniature pressure transducers. The options are discussed by Vinen in these Proceedings [47].

\section{CONCLUSIONS}

It is evident that the theory of QT for $T \rightarrow 0$ has overtaken the experiments and moved far ahead. The study of QT in this range not only brings intrinsic interest of turbulence in a pure superfluid, but also represents a major intellectual challenge. As a consequence of the quantized circulation in superfluids it introduces new physics, e.g. the Kelvin wave cascades. Although adequate tests of the theory require the introduction of new experimen-

\footnotetext{
${ }^{3}$ Designed and built in collaboration with W.F. Vinen, but yet to be tested.
}

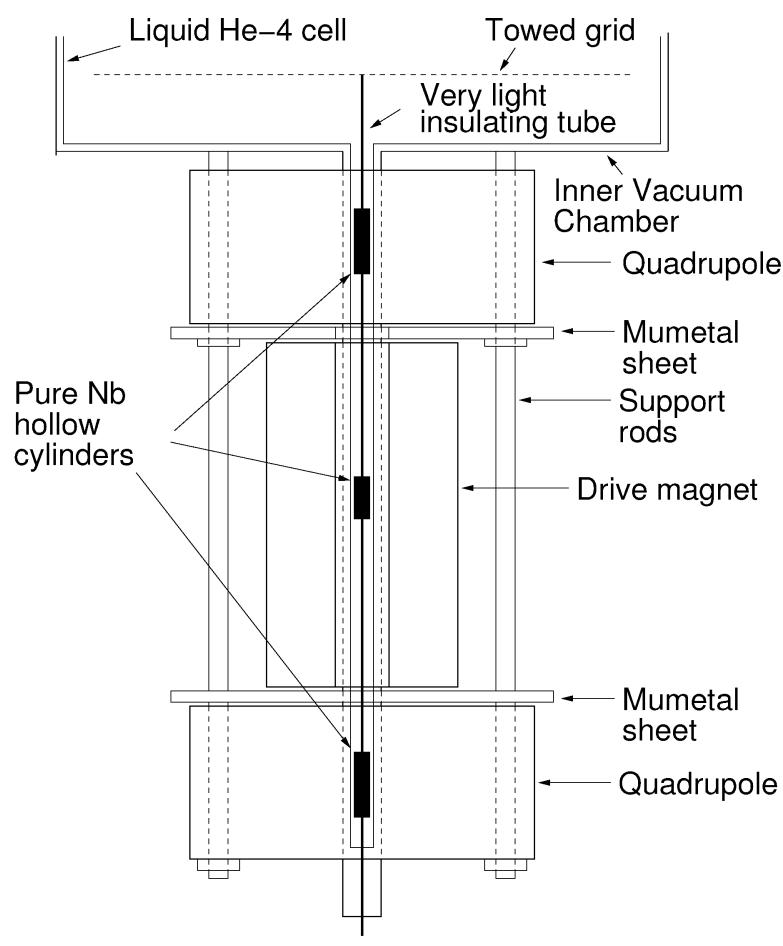

FIGURE 7. Schematic diagram of the linear mechanism being developed for used for creation of well-characterised homogeneous QT at very low temperatures. It uses magnetic bearings and propulsion, and the moving elements should not make any physical contact with static components of the cell.

tal techniques for the creation and detection of QT, there are several experiments under way in different laboratories as well as many promising new ideas ready to be tried. It seems to us that the prospects for rapid progress towards an understanding of QT in the low temperature limit are excellent.

\section{ACKNOWLEDGEMENTS}

We acknowledge valuable discussions with $\mathrm{W} F$ Vinen and G G Ihas and we are grateful to G R Pickett for providing the oscillating loop diagrams in Fig. 5. The work was supported by the Engineering and Physical Sciences Research Council (UK) and by the Ministry of Education of the Czech Republic under research plan MS 002162083.

\section{REFERENCES}

1. Vinen, W. F., and Niemela, J. J., J. Low Temp. Phys., 128, 167-231 (2002).

2. Skrbek, L., Physica C, 404, 354-362 (2004). 
3. Hall, H. E., and Vinen, W. F., Proc. Roy. Soc. (Lond.) A, 238, 204-214 (1956).

4. Vinen, W., Progress in Low Temperature Physics, III, Chapter 1 (1961).

5. Wilks, J., The Properties of Liquid and Solid Helium, Clarendon Press, Oxford, 1967.

6. Batchelor, G. K., The Theory of Homogeneous Turbulence, Cambridge University Press, Cambridge, 1953.

7. Frisch, U., Turbulence, Cambridge University Press, Cambridge, 1995.

8. Landau, L. D., and Lifshitz, E. M., Fluid Mechanics, Butterworth and Heinemann, Oxford, 1987.

9. Maurer, J., and Tabeling, P., Europhys. Lett., 43, 29-34 (1998).

10. Schwarz, K. W., Phys. Rev. B, 38, 2398-2417 (1988).

11. Stalp, S. R., Skrbek, L., and Donnelly, R. J., Phys. Rev. Lett., 82, 4831-4834 (1999).

12. Skrbek, L., Niemela, J. J., and Donnelly, R. J., Phys. Rev. Lett., 85, 2973-2976 (2000).

13. Skrbek, L., Niemela, J. J., and Sreenivasan, K. R., Phys. Rev. E, 64, 067301 (2001).

14. Ellis, T., and McClintock, P. V. E., Phil. Trans. R. Soc. (Lond.) A, 315, 259-300 (1985).

15. Svistunov, B. V., Phys. Rev. B, 52, 3647 (1995).

16. Tsubota, M., Araki, T., and Nemirovskii, S. K., J. Low Temperature Phys., 119, 337-342 (2000).

17. Barenghi, C. F., and Samuels, D., Phys. Rev. Lett., 89, 155302 (2002)

18. Vinen, W. F., Tsubota, M., and Mitani, A., Phys. Rev. Lett., 91, 135301 (2003).

19. Nore, C., Abid, M., and Brachet, M. E., Phys. Rev. Lett., 78, 3896- (1997).

20. Nore, C., Abid, M., and Brachet, M. E., Phys. Fluids, 9, 2644 (1997).

21. Vinen, W. F., Tsubota, M., and Mitani, A., J. Low Temperature Phys., 134, 457-462 (2004).

22. Koplik, J., and Levine, H., Phys. Rev. Lett., 71, 1375 (1993).

23. Kobayashi, M., and Tsubota, M., Phys. Rev. Lett., 94, 065302 (2005).

24. Davis, S. I., Hendry, P. C., and McClintock, P. V. E., Physica B, 280, 43-44 (2000).

25. Morishita, M., Kuroda, T., Sawada, A., and Satoh, T., J. Low Temp. Phys., 76, 387 (1989).

26. Bradley, D. I., Phys. Rev. Lett., 84, 1252 (2000).

27. Yano, H., Handa, A., Nakagawa, H., Obara, K., Ishikawa, O., Hata, T., and Nakagawa, M., J. Low Temperature Phys., 138, 561-566 (2005).

28. Jäger, J., Schuderer, B., and Schoepe, W., Phys. Rev. Lett., 74, 566-569 (1995).

29. Niemetz, M., Kerscher, H., and Schoepe, W., J. Low Temp. Phys, 126, 287-296 (2002).

30. Niemetz, M., and Schoepe, W., J. Low Temperature Phys., 135, 447 (2004).

31. Schoepe, W., Phys. Rev. Lett., 92, 095301 (2004).

32. Bradley, D. I., Clubb, D. O., Fisher, S. N., Guénault, A. M., Haley, R. P., Matthews, C. J., Pickett, G. R., Tsepelin, V., and Zaki, K., Phys. Rev. Lett., 95, 035302 (2005).

33. Nichol, H. A., Skrbek, L., Hendry, P. C., and McClintock, P. V. E., Phys. Rev. Lett., 92, 244501/1-4 (2004).
34. Nichol, H. A., Skrbek, L., Hendry, P. C., and McClintock, P. V. E., Phys. Rev. E, 70, 056307 (2004).

35. Eltsov, V. B., Hosio, J., and Skrbek, L., in preparation (2006).

36. Ellis, T., and McClintock, P. V. E., Phys. Lett. A, 89, 414-416 (1982).

37. Charalambous, D., Hendry, P. C., McClintock, P. V. E., Skrbek, L., and Vinen, W. F., "Vibrating Grid as a Tool for Studying the Flow of Pure He II and its Transition to Turbulence," in these Proceedings.

38. Yano, H., Handa, A., Nakagawa, M., Obara, K., Ishikawa, O., and Hata, T., "Study on the turbulent flow of superfluid ${ }^{4}$ He generated by a vibrating wire," in these Proceedings.

39. Vinen, W. F., Skrbek, L., and Nichol, H. A., J. Low Temperature Phys., 135, 423-445 (2004).

40. Kusmartsev, F. V., Phys. Rev. Lett., 76, 1880-1883 (1996).

41. Awschalom, D. D., and Schwarz, K. W., Phys. Rev. Lett., 52, 49-52 (1984).

42. Finne, A. P., Araki, T., Blaauwgeers, R., and et al, Nature, 424, 1022 (2003).

43. Bradley, D. I., Fisher, S. N., Guénault, A. M., Lowe, M. R., Pickett, G. R., Rahm, A., and Whitehead, R. C. V., Phys. Rev. Lett., 93, 235302 (2004).

44. Honji, H., J. Fluid Mech., 107, 509-520 (1981).

45. de Silva, I. P. D., and Fernando, H. J. S., Phys. of Fluids, 6, 2455-2464 (1994).

46. Ostermeier, R. M., and Glaberson, W. I., J. Low Temp. Phys., 20, 159 (1975).

47. Vinen, W. F., "Quantum turbulence: where do we go from here?," in these Proceedings. 